journal club

\title{
Kein Schutz vor Demenz?
}

Fragestellung: Kann die Entwicklung einer Demenz durch ambulante Kontrolle und Korrektur multipler kardiovaskulärer Risikofaktoren verhindert werden?

Hintergrund: Demenz betrifft aktuell weltweit 36 Millionen Menschen. Die Prävalenz wird wahrscheinlich in den nächsten Jahrzehnten noch ansteigen. WHO und G8-Staaten haben diese Herausforderung erkannt und dazu aufgerufen, Strategien zur Verhinderung von Demenz zu entwerfen. Beobachtungsstudien haben immer wieder gezeigt, dass die typischen kardiovaskulären Risikofaktoren und ein ungesunder Lebensstil mit Demenzen bei Menschen über 65 Jahren assoziiert sind. Populationsbasierte autoptische Studien zeigten, dass neben der Alzheimer-Pathologie vaskuläre Probleme einen bedeutenden Anteil an Demenzen haben. Dies legt nahe, dass es ein Potenzial zur Verhinderung von Demenz gibt, wenn vaskuläre Risikofaktoren behandelt werden. Wenn es randomisierte, kontrollierte Studien zu vaskulären oder Life-style-Risikofaktoren gab, wie zum Hypertonus, zu Inaktivität, ungesundem Essen oder Rauchen, dann wurde aber meist nur ein Faktor isoliert behandelt. Die Resultate waren dann ge-

Moll van Charante EP, Richard E, Eurelings LS et al. Effectiveness of a 6 -year multidomain vascular care intervention to prevent dementia (preDIVA): a cluster-randomised controlled trial. Lancet 2016; 388; $797-805$ mischt und Metaanalysen, zum Beispiel zur antihypertensiven Behandlung zur Demenzprävention, kamen $\mathrm{zu}$ unterschiedlichen Schlussfolgerungen. Diese Diskrepanzen könnten durch Unter- schiede zwischen den Studienpopulationen, zu kurze Beobachtungszeiträume, zu kleine Stichproben und Studienabbrüche erklärt werden. Auch ärztlich begründete Angst vor Nebenwirkungen der antihypertensiven Behandlung und paradoxe Effekte auf die Kognition haben die Entscheidungen zur Blutdrucksenkung bei älteren Menschen erschwert. In der vorliegenden Studie wurden die Effekte einer Kontrolle und gegebenenfalls Korrektur multipler Risikofaktoren bei älteren Menschen untersucht.

Patienten und Methodik: Aus 116 Allgemeinarztpraxen wurden 3.526 Teilnehmer (70 bis 78 Jahre) rekrutiert. 63 Praxen (1.890 Teilnehmer) bildeten die Interventions- und 53 (1.636 Teilnehmer) die Kontrollgruppe. Die Intervention bestand aus der Konsultation einer Pflegeperson in der Praxis alle vier Monate. Dabei wurden die Risikofaktoren erfasst. Alle zwei Jahre wurde Blut entnommen. Auf der Basis der Ergebnisse wurden individuelle Gesundheitsanweisungen mit motivierenden Gesprächstechniken vermittelt. Wenn nötig, wurde eine medikamentöse Behandlung von Hypertonus, Dyslipidämie und Typ-2-Diabetes eingeleitet. Die Kontrollgruppe erhielt eine Standardbehandlung.

Ergebnisse: Nach durchschnittlich 6,7 Jahren hatten in beiden Gruppen 7\% eine Demenz entwickelt. Die körperliche Behinderung war gleich und $16 \%$ waren in beiden Gruppen im Beobachtungszeitraum verstorben.

Schlussfolgerung: Die Korrektur multipler kardiovaskulärer Lebensstilrisikofaktoren reduziert nicht die Demenzhäufigkeit.

\section{- Kommentar von Markus Weih, Nürnberg}

\section{Viel früher mit der Kontrolle beeinflussbarer Risikofaktoren beginnen}

Alois Alzheimer selbst hatte schon auf amyloidartige Veränderungen an zerebralen Arteriolen und Arterien hingewiesen. Dies hat vermutlich durchaus klinische Relevanz: Viele Patienten haben den klinisch typischen Verlauf einer langsam progredienten Alzheimer-Demenz, aber im MRT neben der allgemeinen Atrophie auch deutliche vaskuläre Marklagerläsionen. Die wahrscheinlich hierzu beitragenden kardiovaskulären Risikofaktoren sind hinlänglich bekannt und eigentlich auch beeinflussbar. Angesichts mangelnder Fortschritte in der kausalen Behandlung der Amyloid-Plaque-Pathologie war es damit naheliegend, sich als Demenzprävention in einem allgemeinmedizinischen Setting mit einem umfassenden Ansatz den Risikofaktoren zu nähern. Leider scheiterte dieser wirklich gute Plan der preDIVAStudienärzte aus Amsterdam. Am Studiendesign lag es nicht: Die Stichprobe war ausreichend groß und der Behandlungszeitraum mit über sechs Jahren erfreulich lang. Nur $2 \%$ der immerhin teils sehr alten Teilnehmer gingen verloren und über 100 erreichten in jedem Studienarm eine Demenz als Endpunkt.

Dennoch zeigte sich kein Effekt. Die Erklärung dafür ist eigentlich naheliegend. Die Teilnehmer waren als Kompro- miss, eine Hochrisikopopulation zu erhalten, dann doch zu alt und die Therapie setzte zu spät ein. Sollte sich ein Effekt durch Intervention von Risikofaktoren erzielen lassen, müsste diese wohl viel früher beginnen und länger durchgeführt werden. Dies ist vermutlich in Studien nicht und niemals abzubilden. Fach- und Allgemeinarzt können also den besorgten Kindern von Demenzkranken eingehend ans Herz legen, ihre beeinflussbaren Risikofaktoren schon im mittleren Lebensalter zu kontrollieren und zu behandeln.

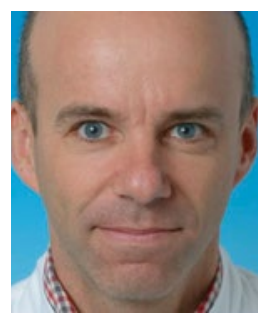

Prof. Dr. med. Markus Weih, Nürnberg

Facharzt für Neurologie, Psychiatrie

und Psychotherapie,

Nervenärztliche Gemeinschaftspraxis

E-Mail:weih@nervenaerzte-

allersbergerstrasse.de 